\title{
Does an enhanced recovery after surgery protocol change costs and outcomes of single-level lumbar microdiscectomy?
}

\author{
Ebru Tarıkçı Kılıç, MD, ${ }^{1}$ Tuncay Demirbilek, MD, ${ }^{2}$ and Sait Naderi, MD² \\ Departments of ${ }^{1}$ Anesthesiology and Perioperative Medicine and ${ }^{2}$ Neurosurgery, Ümraniye Training and Research Hospital, \\ Ümraniye, İstanbul, Turkey
}

OBJECTIVE Enhanced recovery after surgery (ERAS) is a multimodal approach that aims to improve perioperative surgical outcomes. The aim of this study was to evaluate the benefits of ERAS in terms of cost-effectiveness and postoperative outcomes in single-level lumbar microdiscectomy.

METHODS This study was a single-center retrospective comparing costs and outcomes before and after implementation of the ERAS pathway. Data were collected from the electronic medical records of patients who had undergone single-level lumbar microdiscectomy during 2 time periods-during the 2 years preceding implementation of the ERAS pathway (pre-ERAS group) and after implementation of the ERAS pathway (ERAS group). Each group consisted of 60 patients with an American Society of Anesthesiologists (ASA) Physical Status Classification of class 1. Patients were excluded if their physical status was classified as ASA class II-V or if they were younger than 18 years or older than 65. Groups were compared in terms of age, sex, body mass index (BMI), perioperative hemodynamics, operation time, intraoperative blood loss, intraoperative fluid administration, intraoperative opioid administration, time to first oral intake, time to first mobilization, postoperative nausea and vomiting (PONV), difference between preoperative and postoperative visual analog scale (VAS) scores, postoperative analgesic requirements, length of hospital stay, and cost of anesthesia.

RESULTS The ERAS and pre-ERAS groups were comparable with respect to age, sex, and BMI. Operation time, intraoperative blood loss, intraoperative opioid administration, and intraoperative fluid administration were all less in the ERAS group. First oral intake and first mobilization were earlier in the ERAS group. The incidence of PONV was less in the ERAS group. Postoperative analgesic requirements and postoperative VAS scores were significantly less in the ERAS group. The length of hospital stay was found to be shorter in the ERAS group. The ERAS approach was found to be cost-effective.

CONCLUSIONS ERAS had clinical and economic benefits and is associated with improved outcomes in lumbar microdiscectomy. https://thejns.org/doi/abs/10.3171/2019.1.FOCUS18665

KEYWORDS enhanced recovery after surgery; ERAS; lumbar microdiscectomy; cost-effective; economics

$\mathrm{E}$ NHANCED recovery after surgery (ERAS) is a fasttrack multimodal approach that is aimed at improving patient outcomes through all its protocols. It has a synergistic effect on pre-, intra-, and postoperative interventions leading to improvements in perioperative care and overall clinical outcomes. ${ }^{6,7}$ Preoperative education helps to relieve anxiety and allows for earlier return to daily activities. This protocol ends prolonged fasting, mobility limitations, and fluid overload and has gained major popularity by lowering the recovery time, complication rates, and the length of hospital stay. ERAS protocols standardize analgesic and anesthetic regimens and change the traditional surgical pathways rationalizing healthcare resource utilization. . $15,21,22^{2}$

Initially ERAS was introduced in the 1990s for open colorectal surgery but has been investigated in several sur-

ABBREVIATIONS ASA = American Society of Anesthesiologists; $\mathrm{BMI}=$ body mass index; $\mathrm{DBP}=$ diastolic blood pressure; ERAS = enhanced recovery after surgery; HR = heart rate; $\mathrm{PACU}=$ post-anesthetic care unit; $\mathrm{PONV}=$ postoperative nausea and vomiting; $\mathrm{SBP}=$ systolic blood pressure; $\mathrm{S}_{\mathrm{p}} \mathrm{O}_{2}=$ peripheral oxygen saturation; TIVA = total intravenous anesthesia; $T L=$ Turkish Liras; VAS = visual analog scale.

SUBMITTED November 30, 2018. ACCEPTED January 16, 2019.

INCLUDE WHEN CITING DOI: 10.3171/2019.1.FOCUS18665. 
gical specialties including spine surgery. ${ }^{8}$ Spine surgery has lagged behind several other subspecialties in adopting ERAS protocols. ${ }^{13}$

There are still several barriers, including cultural concerns, conventional attitudes, and institutional resistance to change or lack of experience of the surgeons regarding this new approach. Due to the heterogeneity of pathology, variations in the extent of the surgical stress response, and multiple different options for surgical and anesthetic approaches, no unique ERAS pathway has been defined for all spine surgeries.

Although a large number of clinical studies have confirmed the benefits of an ERAS program, the feasibility and effectiveness in the setting of spine surgery are still unknown.

Therefore we aimed to compare the cost-effectiveness and surgical outcomes of single-level lumbar microdiscectomy before and after we instituted an ERAS pathway.

\section{Methods \\ Study Designs}

This was a retrospective study, involving 120 patients with American Society of Anesthesiologists (ASA) Physical Status Classification of class 1, who underwent single-level lumbar microdiscectomy. Data were collected by manual review of the electronic medical record. All procedures were performed in strict accordance with the principles set by the Declaration of Helsinki.

The records of 60 patients who had undergone surgery who were treated just after ERAS implementation (ERAS group) and 60 patients who were treated in the 2 years preceding ERAS implementation (the last 60 treated during that period, pre-ERAS group) were compared in terms of age, sex, body mass index (BMI), perioperative hemodynamics, operation time, intraoperative blood loss, intraoperative fluid administration, intraoperative opioid administration, time to first oral intake, time to first mobilization, postoperative nausea and vomiting (PONV), preoperative-postoperative visual analog scale (VAS) pain scores, postoperative analgesic requirements, length of hospital stay, and cost of anesthesia.

\section{Pre-ERAS Group}

Patients were admitted the day before the operation. The perioperative care and preoperative counseling depended on the surgeon's preference. Prophylactic antibiotic treatment was administered 30 minutes before the patient was taken to the operating room. Thromboembolism prophylaxis and antiemetic prophylaxis were not used. Fasting time for clear fluid was 4 hours and that for solids was 8 hours.

Systolic blood pressure (SBP), diastolic blood pressure (DBP), heart rate (HR), and peripheral oxygen saturation $\left(\mathrm{S}_{\mathrm{p}} \mathrm{O}_{2}\right)$ were monitored throughout the procedure. The time points of these indexes were recorded before induction of anesthesia, after the induction of anesthesia, at 30 minutes into the surgery, and after the completion of the surgery.

General anesthesia was induced using fentanyl 2-4 $\mu \mathrm{g} /$ $\mathrm{kg}$, propofol $2 \mathrm{mg} / \mathrm{kg}$, vecuronium $0.6-0.8 \mathrm{mg} / \mathrm{kg}$, and midazolam $0.1-0.2 \mathrm{mg} / \mathrm{kg}$. After tracheal intubation, the patients were placed in the prone position on the operating table and then anesthesia was maintained via inhalation of $2 \%$ sevoflurane 50\%:50\% oxygen/nitrous oxide. Convective warming was not used. When the breathing pattern was restored the patient was extubated and transferred to the post-anesthetic care unit (PACU). The operation time and total blood loss were recorded. The standard intravenous fluid administration rate was set at $2.5 \mathrm{~L} / 24$ hours. In case of hypotension bolus fluid resuscitation was used. Postoperative oral intake was prohibited. For postoperative pain management intravenous meperidine or morphine substitutes were used. Local infiltrative analgesia into the subcutaneous tissues after closure was not used. Nasogastric tubes or urinary catheters were not used. Patients were discharged when they were able to start daily activities and otherwise ready for discharge.

\section{ERAS Group}

Patients were admitted the day before the operation. Preoperative counseling was performed by anesthesiologists, surgeons, and nurses about the ERAS program for psychological comfort and education. We engaged support from our hospital to schedule the same anesthesiologists, surgeon, and nurses for the ERAS patients wherever possible. Selection was based on their understanding of and willingness to apply ERAS principles. According to our hospital policy we permitted clear fluids up to 2 hours before surgery and solid foods up to 4 hours before surgery. Perioperative fluid management was restricted to avoid overload.

On the day of the operation all patients received antibiotic prophylaxis 30 minutes before the first incision of the operation in compliance with ERAS guidelines. Thromboembolism prophylaxis with low-molecular-weight heparin (injected subcutaneously) and antiemetic prophylaxis of $0.15 \mathrm{mg} / \mathrm{kg}$ ondansetron and $0.2 \mathrm{mg} / \mathrm{kg}$ dexamethasone (injected intravenously) were administered preoperatively. The patients' SBP, DBP, HR, and $\mathrm{S}_{\mathrm{p}} \mathrm{O}_{2}$ were monitored throughout the surgical procedure and the values were recorded at the following time points: before induction of anesthesia, after induction of anesthesia, at 30 minutes after the beginning of the surgery, and after the completion of the surgery. Total intravenous anesthesia (TIVA) was preferentially induced with fentanyl $1 \mu \mathrm{g} / \mathrm{kg}$ and $2 \mathrm{mg} / \mathrm{kg}$ propofol; pure oxygen ventilation (via endotracheal tube) was used during surgery, and anesthesia was maintained using $2-4 \mathrm{mg} / \mathrm{kg} / \mathrm{hr}$ propofol via intravenous micropump until the operation was completed. Convective warming devices were used to achieve normothermia at $36^{\circ} \mathrm{C}$ throughout the operation. When the breathing pattern was restored the patient was extubated and transferred to the PACU. The duration of the operation and total amount of blood loss were recorded. Fluid management was limited to 500 $\mathrm{ml}$ during surgery to achieve euvolemia for all patients. In case of hypotension, vasopressors were administered. For postoperative pain management, a $30-\mathrm{ml}$ bolus containing $0.5 \%$ bupivacaine hydrochloride (Marcaine) was infiltrated into the local subcutaneous tissue after closure and acetaminophen $(1000 \mathrm{mg})$ was administered intravenously. No nasogastric tubes or urinary catheters were utilized. When the patients returned to the regular ward, they were 
TABLE 1. Demographic characteristics and BMI

\begin{tabular}{ccccc}
\hline Characteristic & All Pts $(n=120)$ & Pre-ERAS $(n=60)$ & ERAS $(n=60)$ & $p$ Value \\
\hline Mean age in yrs & $50.12 \pm 6.43$ & $49.80 \pm 6.04$ & $50.43 \pm 6.84$ & $0^{* 691^{*}}$ \\
\hline Sex, $\mathrm{n}(\%)$ & & & $0.360 \dagger$ \\
\hline Female & $65(54.2)$ & $35(58.3)$ & $30(50.0)$ & \\
\hline Male & $55(45.8)$ & $25(41.7)$ & $30(50.0)$ & \\
\hline Mean BMl in $\mathrm{kg} / \mathrm{m}^{2}$ & $28.33 \pm 4.63$ & $28.56 \pm 5.34$ & $28.09 \pm 3.83$ & $0.918^{*}$ \\
\hline
\end{tabular}

Pts = patients

Mean values are given with SDs.

* Student t-test.

$\dagger$ Pearson chi-square test.

encouraged to sit out of the bed for mobilization within 2 hours and oral intake was resumed as soon as possible. An opioid-sparing multimodal approach to pain management was adopted. VAS scores were recorded at 6 and 12 hours after surgery. In patient education, it was emphasized that acetaminophen should be used before an opioid analgesic. Tramadol was available for rescue analgesia. Patients were discharged from the hospital when they had satisfactory recovery with respect to activities of daily living and pain control and were otherwise ready for discharge.

\section{Surgical Technique}

All patients underwent a 1-level microdiscectomy procedure. Patients were positioned prone on a surgical frame in a neutral position. After the patient was positioned, prepped, and draped, C-arm fluoroscopy of the lumbar spine was performed for confirmation of the surgical intervertebral disc level. In the pre-ERAS group, a $2.5-\mathrm{cm}$ skin incision was made, and the fascia was opened in the midline. The skin incision was shorter than $2 \mathrm{~cm}$ in the patients in the ERAS group. A 10-mm-diameter Taylor retractor was used for retraction of paravertebral muscles at the level of the herniated disc. The Taylor retractor was docked using navigation guidance to confirm the level of surgery. Once the retractor was docked, a microscope was brought in and used until the removal of the Taylor retractor. In the pre-ERAS group the microdiscectomy procedure was carried out after removal of the ligamentum flavum, whereas in the ERAS group the microdiscectomy procedure was performed using a ligamentum flavumpreserving technique, as described in the literature. ${ }^{16}$ Subcutaneous closure was performed with 2-0 Vicryl suture. In the ERAS group about $30 \mathrm{ml}$ of $0.5 \%$ bupivacaine hydrochloride was infiltrated into the subcutaneous tissues after closure, as described above. The skin was reapproximated and was sutured with 3-0 Vicryl in pre-ERAS group patients, and with Dermabond and a Steri-Strip in the ERAS group. No Foley catheters or drains of any sort were utilized.

\section{Statistical Analysis}

Data were analyzed using NCSS (2007) and the variables are presented as mean \pm standard deviation (SD) or number. Age, BMI, and perioperative hemodynamic variables were compared between groups using Student t-test.
Continuous data, operation time, intraoperative blood loss, intraoperative fluid administration, and intraoperative opioid requirements were analyzed using Mann-Whitney U-tests, and preoperative-postoperative VAS scores were analyzed using Wilcoxon signed-rank tests. Sex, postoperative analgesic use, and PONV were compared using Pearson chi-square tests. A p value $<0.05$ was considered statistically significant.

\section{Results}

The ERAS group included 60 ASA 1 patients with a mean age of $50.43 \pm 6.84$ years, and the pre-ERAS group included 60 ASA 1 patients with a mean age of $49.80 \pm$ 6.04 years $(p=0.69)$. There were no significant differences between the groups in terms of demographic parameters $(\mathrm{p}>0.5)$ (Table 1).

There was no statistically significant between-groups difference with respect to SBP or DBP before induction of anesthesia, but the SBP and DBP at other time points were significantly lower in the ERAS group (all $p<0.05$ ).

There was no statistically significant between-groups difference with respect to HR or $\mathrm{S}_{\mathrm{p}} \mathrm{O}_{2}($ all $\mathrm{p}>0.05)$ (Table 2).

The mean operation time was $86.42 \pm 18.39$ minutes in the pre-ERAS group and $78.50 \pm 25.20$ minutes in the ERAS group $(p=0.012)$. The mean blood loss was less in the ERAS group than in the pre-ERAS group $(p=0.001)$. Intraoperative fluid administration and intraoperative opioid administration were found to be less in the ERAS group $(p=0.001)$. First postoperative oral intake and first postoperative mobilization were significantly earlier in the ERAS group $(p=0.001)$. The incidence of recorded PONV was less in the ERAS group $(\mathrm{p}=0.001)$. Postoperative analgesic requirements were found to be significantly less in the ERAS group $(p=0.001)$. The length of hospital stay was found to be $30.10 \pm 7.80$ hours in the pre-ERAS group and $26.52 \pm 5.16$ hours in the ERAS group ( $\mathrm{p}=$ 0.001 ). The cost of anesthesia was found to be $270.42 \pm$ 87.16 Turkish Liras (TL) in the pre-ERAS group, but only $73.00 \pm 24.93 \mathrm{TL}$ in the ERAS group $(\mathrm{p}=0.001)$ The cost of surgery was found to be $1991.67 \pm 67.12$ TL in the preERAS group and $1258.67 \pm 39.89 \mathrm{TL}$ in the ERAS group $(\mathrm{p}=0.001)$ (Table 3). Postoperative VAS scores were found to be significantly lower in the ERAS group ( $\mathrm{p}=$ 0.001) (Table 4). 
TABLE 2. Vital signs of patients in the pre-ERAS and ERAS groups at different time points

\begin{tabular}{|c|c|c|c|c|c|}
\hline Parameter \& Group & Before Induction of Anesthesia & After Induction of Anesthesia & 30 Mins Into Op & At End of the Op & p Value* \\
\hline $\mathrm{SBP}(\mathrm{mm} \mathrm{Hg})$ & & & & & 0.039 \\
\hline Pre-ERAS & $141.60 \pm 16.30$ & $136.60 \pm 15.40$ & $132.20 \pm 18.70$ & $117.60 \pm 14.50$ & \\
\hline ERAS & $140.40 \pm 20.90$ & $116.60 \pm 25.80$ & $112.80 \pm 23.80$ & $135.70 \pm 8.50$ & \\
\hline $\mathrm{DBP}(\mathrm{mm} \mathrm{Hg})$ & & & & & 0.035 \\
\hline Pre-ERAS & $89.10 \pm 9.40$ & $83.20 \pm 8.70$ & $77.30 \pm 10.60$ & $72.40 \pm 15.30$ & \\
\hline ERAS & $74.20 \pm 15.6$ & $64.40 \pm 13.60$ & $66.80 \pm 7.40$ & $68.80 \pm 10.30$ & \\
\hline $\mathrm{HR}$ (bpm) & & & & & 0.670 \\
\hline Pre-ERAS & $74.40 \pm 15.50$ & $76.30 \pm 17.70$ & $78.40 \pm 16.40$ & $73.40 \pm 16.70$ & \\
\hline ERAS & $73.60 \pm 18.50$ & $78.50 \pm 15.70$ & $75.30 \pm 13.10$ & $70.70 \pm 19.90$ & \\
\hline $\mathrm{S}_{\mathrm{p}} \mathrm{O}_{2}(\%)$ & & & & & 0.917 \\
\hline Pre-ERAS & $92.50 \pm 4.10$ & $93.40 \pm 4.90$ & $93.70 \pm 4.80$ & $93.80 \pm 4.10$ & \\
\hline ERAS & $92.60 \pm 3.80$ & $92.90 \pm 8.20$ & $93.30 \pm 3.50$ & $93.80 \pm 7.60$ & \\
\hline
\end{tabular}

${ }^{*}$ Student t-test.

\section{Discussion}

The success achieved with the use of ERAS in many surgical specialties suggests that similar benefits can be achieved in spine surgery. ${ }^{9}$ Our study highlights the positive perioperative outcomes in patients undergoing lumbar microdiscectomy. The key challenge is the ERAS pathway itself, with the operative team's compliance with the protocols rather than the surgical approach as the main determinant of good outcomes. ${ }^{4}$

In this paper we provide a first full description of our ERAS protocol tailored for lumbar microdiscectomy and demonstrate its association with short operation time, less blood loss, lower pain scores, shorter length of hospital stay, and lower cost.

Our enhanced recovery program included patient participation education, appropriate antibiotic administration, thromboembolism prophylaxis, opioid-sparing anesthetic management with TIVA, temperature management, fluid limitations, PONV prophylaxis, pain management, and implementation of a minimally invasive surgical technique. In the postoperative period early mobilization and early diet advancements were strongly supported by our ERAS team.

The use of TIVA minimized the stress response, avoid-

TABLE 3. Intraoperative and postoperative outcomes

\begin{tabular}{|c|c|c|c|c|}
\hline Variable & All Pts $(n=120)$ & Pre-ERAS $(n=60)$ & ERAS $(n=60)$ & $p$ Value \\
\hline Op time in mins & $82.46 \pm 22.32$ & $86.42 \pm 18.39$ & $78.50 \pm 25.20$ & $0.012^{*} \dagger$ \\
\hline Intraop blood loss in $\mathrm{ml}$ & $140.42 \pm 67.44$ & $187.67 \pm 47.37$ & $93.17 \pm 48.89$ & $0.001^{* *} \dagger$ \\
\hline Intraop fluid admin in ml & $1354.5 \pm 765.79$ & $2044.1 \pm 401.38$ & $665.0 \pm 233.49$ & $0.001^{* *} \dagger$ \\
\hline Intraop opioid admin in $\mu \mathrm{g}$ & $98.96 \pm 51.69$ & $147.92 \pm 22.69$ & 50 & $0.001^{* *} \dagger$ \\
\hline Time to 1st oral intake in hrs & $3.89 \pm 1.42$ & $4.90 \pm 1.08$ & $2.88 \pm 0.92$ & $0.001^{* *} \dagger$ \\
\hline Time to 1st mobilization in hrs & $5.65 \pm 2.36$ & $7.20 \pm 2.33$ & $4.10 \pm 0.95$ & $0.001^{* *} \dagger$ \\
\hline PONV, n (\%) & & & & $0.001^{* *} \ddagger$ \\
\hline No & $73(60.8)$ & $22(36.7)$ & $51(85.0)$ & \\
\hline Yes & $47(39.2)$ & $38(63.3)$ & $9(15.0)$ & \\
\hline Postop analgesic required, $\mathrm{n}(\%)$ & & & & $0.001^{* *} \ddagger$ \\
\hline No & $52(43.3)$ & $0(0)$ & $52(86.7)$ & \\
\hline Yes & $68(56.7)$ & $60(100)$ & $8(13.3)$ & \\
\hline Length of hospital stay in hrs & $28.31 \pm 6.83$ & $30.10 \pm 7.80$ & $26.52 \pm 5.16$ & $0.001^{* *} \dagger$ \\
\hline Cost of anesthesia in TL & $171.71 \pm 117.9$ & $270.42 \pm 87.16$ & $73.00 \pm 24.93$ & $0.001^{* *} \dagger$ \\
\hline Cost of op in TL & $1625.17 \pm 372.12$ & $1991.67 \pm 67.12$ & $1258.67 \pm 39.89$ & $0.001^{* *} \dagger$ \\
\hline \multicolumn{5}{|c|}{$\begin{array}{l}\text { Admin }=\text { administration; } P O N V=\text { postoperative nause and vomiting; } T L=\text { Turkish Liras. } \\
\text { Data are presented as mean } \pm \text { SD unless otherwise indicated. } \\
{ }^{*} p<0.05 \text {. } \\
{ }^{* *} p<0.01 \text {. } \\
\dagger \text { Mann-Whitney U-test. } \\
\ddagger \text { Pearson chi-square test. }\end{array}$} \\
\hline
\end{tabular}


TABLE 4. Change in VAS scores

\begin{tabular}{|c|c|c|c|c|}
\hline & $\begin{array}{c}\text { All Pts } \\
(n=120)\end{array}$ & $\begin{array}{c}\text { Pre-ERAS } \\
(n=60)\end{array}$ & $\begin{array}{l}\text { ERAS } \\
(n=60)\end{array}$ & $\begin{array}{c}p \\
\text { Value* }^{*}\end{array}$ \\
\hline $\begin{array}{l}\text { Preop VAS } \\
\text { score }\end{array}$ & $7.73 \pm 1.32$ & $7.58 \pm 1.14$ & $7.87 \pm 1.48$ & 0.101 \\
\hline $\begin{array}{l}\text { VAS score at } 6 \\
\text { hrs postop }\end{array}$ & $2.86 \pm 1.66$ & $4.03 \pm 0.88$ & $1.68 \pm 1.40$ & $0.001^{* *}$ \\
\hline $\begin{array}{c}\text { VAS score at } 12 \\
\text { hrs postop }\end{array}$ & $3.53 \pm 1.77$ & $3.08 \pm 0.90$ & $1.12 \pm 0.80$ & $0.001^{* *}$ \\
\hline $\begin{array}{l}\text { Change in VAS } \\
\text { score } †\end{array}$ & $-4.87 \pm 1.87$ & $-3.55 \pm 1.28$ & $-6.18 \pm 1.37$ & $0.001^{* *}$ \\
\hline
\end{tabular}

ing the consistent inhalation/opioid-based anesthetic regimen. Selecting techniques with a view to reducing the physiological response to surgery is one of the ERAS goals, and administering any sedation agent can result in undesirable effects. ${ }^{11,12}$ Additionally, TIVA - with its ease of titration-lowers the risk of airway problems, while facilitating appropriate surgical conditions. Determining the best mode of general anesthesia or regional anesthesia requires additional study of ERAS protocols for spine surgery. ${ }^{1}$

Bupivacaine hydrochloride was used for local infiltration analgesia. Cost-benefit analysis supported its use. Many studies have demonstrated bupivacaine's benefits in reducing postoperative pain, similar to our findings. Moreover, routine use of bupivacaine hydrochloride may be applicable in a variety of practice settings. ${ }^{10,17}$

Many studies have demonstrated the benefits of NSAID use in the postoperative period with respect to decreasing opioid use and opioid-related side effects. ${ }^{3}$ Sedation, constipation, ileus, and respiratory depression are serious opioid-related side effects that can extend the recovery period and hospital stay. 3,18

We chose a minimally invasive surgical technique for our ERAS pathway. Many systematic reviews and studies suggest minimally invasive approaches for lumbar microdiscectomies due to the associated benefits, including reductions in blood loss, pain, operation time, duration of the recovery period, hospital stay, and costs. ${ }^{19}$ Accordingly we described the development of and early experience with an evidence-based ERAS pathway for minimally invasive lumbar microdiscectomy procedures. Minimally invasive techniques directly minimize the biochemical stress response, and the targeted hypotension and restricted intraoperative fluid administration of ERAS protocols minimize intraoperative blood loss. Advances in technology, many improvements in surgical specialties, and the minimally invasive techniques all decrease perioperative morbidity.

Wang et al. demonstrated the efficacy and reliability of an ERAS pathway by using a minimally invasive surgical technique for spinal fusions. Sedation with ketofol (combination of ketamine and propofol) was used instead of general endotracheal anesthesia. Tissue trauma was mini- mized with 8-mm incisions. A combination of endoscopic decompression, cage deployment prior to screw placement, and long-acting liposomal bupivacaine infiltrative anesthesia were performed without general endotracheal anesthesia. Similar to our results, the results of Wang and colleagues showed decreased operative time, blood loss, hospital length of stay, and cost of hospitalization in patients treated with an ERAS protocol. ${ }^{21}$

Grasu et al. conducted a retrospective cohort study of spine surgery in patients with metastatic tumors. They described the implementation of ERAS and compared 41 patients who participated in ERAS with 56 patients who underwent surgery before ERAS implementation. The ERAS group had better pain scores with less opioid consumption. ${ }^{5}$

In another study of ERAS in spine surgery, Wainwright et al. reported that ERAS intervention accelerated recovery and provided improved long-term outcomes in major spine surgeries. ${ }^{20}$ Mathiesen et al. studied multimodal pain control and found improved mobilization and less opioid consumption with this approach to pain management after multilevel spine surgery. ${ }^{14}$

Although our study had a small sample size and our determination of patients' readiness for discharge is somewhat subjective and was not easy to capture, we did find that the ERAS group had a significantly shorter length of hospital stay. This result can be explained by 2 factors: shorter operative time and better pain management.

We used institutional accounting data for cost analysis. Our results supported the evidence that ERAS not only provides preoperative benefits but also decreases healthcare costs. The current analysis included assessment of anesthetic and surgery costs and, as expected, ERAS was found to be cost-effective. Randomized controlled trials with the intention of evaluating all associated costs are needed to comprehensively assess the benefits of ERAS in spine surgeries.

Although this study was retrospective, the ERAS and pre-ERAS cohorts were similar in sample size, demographics, ASA classification, and surgery type, allowing for comparison between the groups. Given the short duration of data collection reported here, further initiatives are needed.

\section{Conclusions}

Our report shows that the adoption of a comprehensive ERAS program in spine surgery is beneficial and the program is simple to apply, but we believe that there is a need for international guidelines in order to standardize the comparisons for further improvements of the outcome and care of the patients undergoing spine surgeries.

\section{References}

1. Bekker A, Haile M, Kline R, Didehvar S, Babu R, Martiniuk $F$, et al: The effect of intraoperative infusion of dexmedetomidine on the quality of recovery after major spinal surgery. J Neurosurg Anesthesiol 25:16-24, 2013

2. Carli F: Physiologic considerations of Enhanced Recovery After Surgery (ERAS) programs: implications of the stress response. Can J Anaesth 62:110-119, 2015

3. Dunn LK, Durieux ME, Nemergut EC: Non-opioid analge- 
sics: Novel approaches to perioperative analgesia for major spine surgery. Best Pract Res Clin Anaesthesiol 30:79-89, 2016

4. Grant MC, Hanna A, Benson A, Hobson D, Wu CL, Yuan CT, et al: Dedicated operating room teams and clinical outcomes in an Enhanced Recovery after Surgery Pathway for Colorectal Surgery. J Am Coll Surg 226:267-276, 2018

5. Grasu RM, Cata JP, Dang AQ, Tatsui CE, Rhines LD, Hagan KB, et al: Implementation of an Enhanced Recovery After Spine Surgery program at a large cancer center: a preliminary analysis. J Neurosurg Spine 29:588-598, 2018

6. Greco M, Capretti G, Beretta L, Gemma M, Pecorelli N, Braga M: Enhanced recovery program in colorectal surgery: a meta-analysis of randomized controlled trials. World J Surg 38:1531-1541, 2014

7. Gustafsson UO, Scott MJ, Schwenk W, Demartines N, Roulin D, Francis N, et al: Guidelines for perioperative care in elective colonic surgery: Enhanced Recovery After Surgery $\left(\right.$ ERAS $\left.^{\circledR}\right)$ Society recommendations. World J Surg 37:259284, 2013

8. Joshi GP, Bonnet F, Kehlet H: Evidence-based postoperative pain management after laparoscopic colorectal surgery. Colorectal Dis 15:146-155, 2013

9. Kehlet H, Wilmore DW: Evidence-based surgical care and the evolution of fast-track surgery. Ann Surg 248:189-198, 2008

10. Kim J, Burke SM, Kryzanski JT, Roberts RJ, Roguski M, $\mathrm{Qu} \mathrm{E}$, et al: The role of liposomal bupivacaine in reduction of postoperative pain after transforaminal lumbar interbody fusion: a clinical study. World Neurosurg 91:460-467, 2016

11. Kim KH: Safe sedation and hypnosis using dexmedetomidine for minimally invasive spine surgery in a prone position. Korean J Pain 27:313-320, 2014

12. Kumar G, Stendall C, Mistry R, Gurusamy K, Walker D: A comparison of total intravenous anaesthesia using propofol with sevoflurane or desflurane in ambulatory surgery: systematic review and meta-analysis. Anaesthesia 69:11381150,2014

13. Maessen J, Dejong CH, Hausel J, Nygren J, Lassen K, Andersen J, et al: A protocol is not enough to implement an enhanced recovery programme for colorectal resection. Br J Surg 94:224-231, 2007

14. Mathiesen O, Dahl B, Thomsen BA, Kitter B, Sonne N, Dahl JB, et al: A comprehensive multimodal pain treatment reduces opioid consumption after multilevel spine surgery. Eur Spine J 22:2089-2096, 2013

15. Melnyk M, Casey RG, Black P, Koupparis AJ: Enhanced recovery after surgery (ERAS) protocols: Time to change practice? Can Urol Assoc J 5:342-348, 2011

16. Ozer AF, Oktenoglu T, Sasani M, Bozkus H, Canbulat N, Karaarslan E, et al: Preserving the ligamentum flavum in lumbar discectomy: a new technique that prevents scar tissue formation in the first 6 months postsurgery. Neurosurgery 59 (1 Suppl 1):ONS126-ONS133, 2006

17. Puffer RC, Tou K, Winkel RE, Bydon M, Currier B, Freedman BA: Liposomal bupivacaine incisional injection in single-level lumbar spine surgery. Spine J 16:1305-1308, 2016

18. Rivkin A, Rivkin MA: Perioperative nonopioid agents for pain control in spinal surgery. Am J Health Syst Pharm 71:1845-1857, 2014

19. Sarin A, Litonius ES, Naidu R, Yost CS, Varma MG, Chen LL: Successful implementation of an Enhanced Recovery After Surgery program shortens length of stay and improves postoperative pain, and bowel and bladder function after colorectal surgery. BMC Anesthesiol 16:55, 2016

20. Wainwright TW, Immins T, Middleton RG: Enhanced recovery after surgery (ERAS) and its applicability for major spine surgery. Best Pract Res Clin Anaesthesiol 30:91-102, 2016

21. Wang MY, Chang PY, Grossman J: Development of an Enhanced Recovery After Surgery (ERAS) approach for lumbar spinal fusion. J Neurosurg Spine 26:411-418, 2017

22. Yamada T, Hayashi T, Cho H, Yoshikawa T, Taniguchi H, Fukushima R, et al: Usefulness of enhanced recovery after surgery protocol as compared with conventional perioperative care in gastric surgery. Gastric Cancer 15:34-41, 2012

\section{Disclosures}

The authors report no conflict of interest concerning the materials or methods used in this study or the findings specified in this paper.

\section{Author Contributions}

Conception and design: Naderi. Acquisition of data: Tarıkçı Kılıç. Analysis and interpretation of data: Tarıkçı Kılıç, Demirbilek. Critically revising the article: Naderi. Statistical analysis: Tarıkçı Kılıç.

\section{Correspondence}

Ebru Tarıkçı Kılıç: Ümraniye Training and Research Hospital, Ümraniye, İstanbul, Turkey. ebru.tarkc@yahoo.com. 